Revista de Psicología Vol. 30 (2), 2012 (ISSN 0254-9247)

\title{
Apoyo social y conductas sexuales de riesgo en adolescentes del municipio de Lebrija-Santander
}

\author{
Linda Teresa Orcasita ${ }^{1}$ \\ Pontificia Universidad Javeriana-Seccional Cali (Colombia) \\ Ana Fernanda Uribe ${ }^{2}$, Laura Patricia Castellanos ${ }^{3}$, Marisol Gutiérrez Rodríguez ${ }^{4}$
Universidad Pontificia Bolivariana Bucaramanga (Colombia)
}

La presente investigación evalúa el apoyo social y las conductas sexuales de riesgo presentes en adolescentes de una institución educativa del municipio de Lebrija-Santander. La muestra se conformó por 359 sujetos con edades entre los 12 y 18 años; es una investigación de carácter no experimental de tipo descriptivo-correlacional. Se utilizaron una serie de cuestionarios: Conductas de Riesgo de Vigilancia (YRBS), Cuestionario MOS y finalmente el APGAR Familiar. Los resultados muestran que los adolescentes que han iniciado su vida sexual presentan conductas sexuales de riesgo, las más frecuentes son: la no utilización del preservativo, el consumo de bebidas alcohólicas y el inicio de la vida sexual a edades tempranas. Asimismo los jóvenes que han iniciado su vida sexual son quienes perciben un menor apoyo tanto emocional como instrumental por parte de su principal red de apoyo (la familia). Se concluye sobre la necesidad de fortalecer las redes de apoyo de los adolescentes, debido a que están directamente relacionadas con conductas sexuales de riesgo adoptadas por esta población, es importante la participación no solo de los jóvenes sino también la vinculación de padres de familia, instituciones educativas, docentes, entre otros, en los programas de salud sexual y reproductiva diseńados para los adolescentes.

Palabras clave: adolescentes, apoyo social, redes de apoyo social, conductas sexuales de riesgo y familia

1 Magíster en Psicología y docente de la Pontificia Universidad Católica Javeriana Seccional Cali-Colombia. Grupo de investigación Psicología Clínica y de la Salud. Correo electrónico: ltorcasita@javerianacali.edu.co

2 Doctora en Psicología Clínica y de la Salud y Directora de la Facultad de Psicología Universidad Pontificia Bolivariana-Bucaramanga. Grupo de investigación Psicología Clínica y de la Salud. Correo electrónico: ana.uribe@gmail.com

3 Psicóloga. Universidad Pontificia Bolivariana-Bucaramanga. Grupo de investigación Psicología Clínica y de la Salud. Correo electrónico: laura-castellanos@hotmail.com

4 Psicóloga. Universidad Pontificia Bolivariana-Bucaramanga. Grupo de investigación Psicología Clínica y de la Salud. Correo electrónico: magu490@hotmail.com 


\section{Social support and at risk sexual behavior in adolescents of Lebrija-Santander}

This study examined social support and sexual risk behavior in adolescents attending an educational institution in the municipality of Lebrija Santander. The sample involves 359 teenagers aged 12-18. This is a non-experimental descriptive-correlational study. Measures included a series of questionnaires, involving Risk Behavior Surveillance (YRBS), and the MOS Questionnaire Family APGAR. Results show that teens who started their sexual life, are at risk of sexual behaviors such as: non-condom use, alcohol consumption and the onset of sexual life at an early age. Also young people who have started their sexual life are those who perceive less emotional and instrumental support from their primary support network (family). It concludes on the need to strengthen support networks of adolescents, because they are directly related to sexual risk behavior is taken by people, is important to involve not only young people but also the involvement of parents, institutions education, teachers, among others, programs designed sexual and reproductive health for adolescents.

Keywords: adolescents, social support, social support networks, risk sex behaviors and family 
En el mundo existen 33.3 millones de personas que viven con el VIH, de las cuales 22.5 millones están en África Subsahariana (ONUSIDA 2010). En Colombia anualmente hay un crecimiento del número de casos notificados de VIH, SIDA, los cuales han sido 71509 desde 1983 hasta el año 2009, donde la edad promedio de personas infectadas abarca entre los 25 y 35 años (UNGASS, 2010). En Santander según la Encuesta Nacional de Demografía y Salud (ENDS, 2010) se demuestra que un 33 por ciento de las mujeres entre 15 y 49 años, señaló haberse realizado la prueba para detectar el VIH y un 3 por ciento de ellas señaló haber tenido una Infección de Trasmisión Sexual (ITS). El 46 por ciento de las mujeres en edad fértil sabe que el VIH se puede transmitir de la madre al hijo durante la lactancia, un 14 por ciento de las mujeres de Santander no conoce acerca de las Infecciones de Transmisión Sexual (ITS). De la misma forma, durante el 2009 se registraron 327 nuevos casos de personas infectadas de VIH, donde en comparación al 2008 aumentó el número de personas con el diagnóstico. Un 31\% muestra un predominio en los hombres; $74 \%$ de los casos fueron registrados en el área metropolitana y un 65\% de ellos en la ciudad de Bucaramanga 30.2\% por cada 100000 habitantes con una edad promedio menor o igual a los 34 años (Observatorio Salud Pública de Santander, 2010).

Por otra parte, en el estudio sobre factores de riesgo frente al VIH/ SIDA en adolescentes colombianos, se identificó que en una muestra de 978 adolescentes de ciudades como Bogotá, Cali, Manizales, Pasto y Armenia, se presenta diferencias significativas con respecto a los conocimientos correctos que tienen sobre el VIH/SIDA. Además se encontró que más del $60 \%$ de los adolescentes afirman haber recibido información sobre transmisión y prevención del VIH, principalmente de programas de televisión y charlas en el colegio, sin embargo dicha información aún no ha incidido de manera suficiente, ya que se siguen 
presentando conductas sexuales de riesgo, lo que lleva a pensar que la información ofrecida a esta población requiere profundizar de manera amplia desde otros abordajes que integren las necesidades de los adolescentes (Uribe, Orcasita \& Vergara, 2010).

La adolescencia es una etapa del ciclo de vida que implica una serie de cambios y desafíos evolutivos a nivel emocional, físico, biológico y social; además de la definición de su identidad y la responsabilidad en la toma de decisiones (Musitu \& Cava, 2003; Uribe \& Orcasita, 2010). Por ello, la influencia de los padres, hermanos, amigos mayores, pares, vecinos y compañeros, entre otros, es fundamental durante esta etapa del ciclo de vida puesto que estas redes de apoyo pueden tener efectos positivos o negativos sobre dicha población (Barrón, 1999 citado por Uribe \& Orcasita, 2010).

De acuerdo con las estadísticas y reportes se ha identificado que el inicio de las relaciones sexuales tempranas caracterizadas por la ausencia de medidas de protección y múltiples parejas sexuales, aumentan el riesgo de contraer alguna ITS o VIH/SIDA, lo cual permiten describir a los adolescentes como una población vulnerable ante el aumento cada vez mayor de estas prácticas sin medidas preventivas (Campo, Silva, Meneses, Castillo \& Navarrete, 2004). Estos autores afirman en una investigación hecha en la ciudad de Bucaramanga, que de una población de 122 jóvenes, el 75\% de los estudiantes encuestados afirmaron haber sostenido relaciones sexuales antes de los 16 ańos. Por otra parte en otro estudio sobre consumo de alcohol y conducta sexual de riesgo en adolescentes, aplicado en la ciudad de México a 1000 jóvenes, se detectó que el $30.5 \%$ de la población inició su vida sexual y con una edad promedio de 15.33 ańos, de estos el $8.7 \%$ afirmaron que nunca han usado preservativo y $4.5 \%$ casi nunca lo utilizan (Palacios, Bravo \& Andrade, 2007). Es así como Avendaño y Barra (2008) han considerado después de analizar estudios con diversas enfermedades presentes en los adolescentes que el apoyo social proporcionado por las redes sociales tendría influencia sobre el bienestar, la salud y la calidad de vida de los jóvenes. Es primordial evaluar las redes de apoyo, determinar qué muestran y qué ofrecen estas a los adolescentes y la influencia que 
tienen en la prevención o no de su salud sexual y reproductiva, además de la relación con aquellas conductas que el joven emite.

Por consiguiente, debido a que las investigaciones se centran en estudiar la presencia del apoyo social en la vida adulta; un estudio realizado por Cañellas et al. (2000) identificaron las conductas sexuales de riesgo en hombres con prácticas homosexuales y bisexuales en la comunidad de Madrid. Asimismo, Belzaa et al. (2004) realizaron una investigación orientada a la caracterización de las condiciones sociolaborales, conductas de riesgo y prevalencia de infecciones de transmisión sexual en mujeres inmigrantes. Teniendo en cuenta que existen pocos estudios sobre conductas sexuales de riesgo en los adolescentes de Bucaramanga y su área metropolitana, se hace necesario investigar el tipo de apoyo social percibido por los adolescentes a lo largo de su desarrollo y la influencia sobre las conductas sexuales de los adolescentes.

Las personas, sin importar su edad, procedencia, cultura, religión o característica socioeconómica, presentan necesidades no solo de tipo físico sino también emocional y afectivo, puesto que una de las características del ser humano es la necesidad de estar en constante interrelación con el medio que lo rodea, debido a que este proceso permite generar recursos de apoyo para enfrentar las situaciones de riesgo que se presenten, es decir que el proceso de interrelación interviene de manera positiva o negativa en el bienestar, la salud y la calidad de vida de las personas (Orcasita \& Uribe, 2010).

Durante los últimos ańos el concepto de apoyo social, ha generado diversas investigaciones debido a la incidencia que tiene en el desarrollo biopsicosocial de los individuos. Juárez, Valdez y Hernández (2001) sostienen que el apoyo social es un conjunto conformado por las redes sociales, las cuales están encargadas de "dar y recibir", es decir, es un sistema de reciprocidad e intercambio de bienes y/o servicios. Morales, Cerezo, Fernández, Infante y Trianes (2009), describen al apoyo social como provisiones instrumentales y/o expresivas, reales y percibidas, aportadas por la comunidad, las redes sociales y los amigos íntimos. Entre tanto Musitu y Cava (2003), seńalan que el apoyo social es uno de los principales recursos intrínsecos y extrínsecos con que cuenta 
el individuo; dichos recursos son otorgados por los integrantes de las redes sociales que posee cada individuo; los cuales pueden ser de tipo emocional, material, informacional o de compañía.

Teniendo en cuenta lo establecido por Méndez y Barra (2008) el concepto de apoyo social está asociado a la valoración que una persona tiene sobre su red social (conformada por la familia, la pareja, los amigos, las instituciones, la comunidad, entre otras) y los recursos que esta le provea. Para estos autores el apoyo social cuenta con funciones principales en las siguientes dimensiones: (a) Apoyo emocional, la cual hace referencia a la posibilidad de compartir sentimientos, pensamientos, afectos, reconocimiento, escucha, incidiendo favorablemente en el auto estima y desempeño de los individuos; (b) Cognitivos, se refiere a la guía o consejos, intercambio de experiencias e Información interviniendo en la creación de estrategias de afrontamiento emocional y conductual ante las diferentes situaciones que las personas deban afrontar; y (c) Apoyo instrumental, conformado por el soporte material (dinero, alojamiento, vivienda, comida, ropa) o de servicios (cuidado, transporte, estudios y labores del hogar) que recibe un individuo de su red de apoyo.

Por otro lado, Barrón (1996) distingue los siguientes aspectos del apoyo social: el primero de ellos corresponde a los niveles de análisis, que describen la ayuda accesible a un sujeto, a través de los lazos sociales de otros sujetos, grupos y comunidad global. El segundo aspecto esta vinculado con aquellas personas o sistemas que usualmente forman las redes de apoyo social de los individuos (Barrón, 1996), ya sea por vinculación directa o indirecta. Siendo parte de estos sistemas de redes se encuentran las instituciones religiosas, el sistema de salud, el grupo laboral, y marcadamente la familia, los amigos y el colegio como primeras instancias de socialización de todo sujeto.

Acorde con los datos arrojados en la investigación de Martin y Dávila (2008), Redes de apoyo social y adaptación de los menores en el acogimiento residencial, se precisó que para los adolescentes es fundamental contar con una buena red de apoyo, para superar de manera acertada las situaciones negativas que se pudieran presentar. Dentro del 
proceso de investigación se estableció que para los adolescentes sus principales fuentes de apoyo están conformadas por la madre y los amigos. Una de las herramientas intrínsecas que direcciona las redes de apoyo es la autorregulación, autoconcepto y autoeficacia, estas competencias se desarrollan principalmente en la familia nuclear y posteriormente se fortalecen adecuada o inadecuadamente en el proceso de interacción social e individualización personal. Es así como el fortalecimiento de competencias como autorregulación y autoconcepto está vinculado con el grado de vulnerabilidad o fortaleza, que cuenta el individuo para asumir los cambios que demandan las transiciones propias del desarrollo y la capacidad de adaptación que demanda la sociedad (Avendaño \& Barra, 2008).

De acuerdo con Minuchin (1983), la familia es percibida como un grupo natural que en el transcurso del tiempo diseña pautas de interacción, las cuales establecen la estructura que rige el funcionamiento y roles de los integrantes de la familia, optimizando el proceso de individualización de cada persona. La familia cumple una función que va más allá de garantizar la supervivencia física de sus miembros, asegurando la integración sociocultural de estos a los escenarios y hábitats donde se desenvuelven como personas. La sociedad y la familia tienen una relación bidireccional, puesto que aportan un conjunto de premisas socioculturales, condiciones económicas, normas y valores, que indiscutiblemente, demarcan la manera en que los miembros de la familia pueden relacionarse con los otros miembros de la sociedad (Valdés, 2007); la familia también, es considerada como la unidad básica de salud, encargada de educar con límites y asimismo ejercer autoridad afectiva, compartida y responsable brindando factores protectores (Castellano, 2005 citado por Orcasita \& Uribe, 2010). Andersen, (1999) expresa que la familia como concepto abstracto no existe, pero aun así es posible afirmar que la familia es un sistema de relaciones de parentesco (no necesariamente implica consanguinidad) reguladas de manera muy diferente entre las distintas culturas. Estas relaciones giran en torno a vínculos afectivos entre sus miembros, las cuales son expresadas por medio de alianzas entre los integrantes. 
Para Fernández (2000) la institución educativa también se concentra como una red de apoyo en la existencia de los individuos, pues determina en buena medida las normas que en el futuro serán concluyentes para su vida, discrimina en la realidad del ser lo individual, lo grupal, lo interpersonal, lo organizacional y lo social, vincula al sujeto con sus pares y potenciales amigos, y además generalmente después de la familia, se convierte en la instancia de la cual se espera mayor apoyo especialmente en la niñez y la adolescencia. Las instituciones educativas no solo transmiten conocimientos, sino que también ofrecen pautas para acceder a la conciencia de la individualización, en este sentido, los centros educativos ofrecen un funcionamiento basado en procesos de reflexión y evaluación. Esta formación, permite al mismo tiempo seguridad psicológica y apoyo para mejorar la identificación de las problemáticas y búsqueda de soluciones.

Otra fuente de apoyo importante durante la adolescencia son los amigos, Pardo et al. (2004) citado por Orcasita y Uribe (2010) afirma que el grupo de pares brinda la posibilidad de tomar decisiones junto a sus compañeros, tener poder y seguridad, proporciona prestigio y libertad, constituyéndose en un importante modelo de referencia, en el cual existen emociones, ideas, ambiciones y expectativas que discrepan a los adolescentes con los valores y principios de los adultos. Por ello las entidades educativas no solo son una institución de apoyo en momentos de necesidad, sino también a lo largo de todo el proceso formativo de sus estudiantes y de ser manejado correctamente dicho apoyo, traerá consigo beneficios significativos para las personas que hacen parte de la institución.

Según Barrón (1996) como parte del apoyo social, las redes sociales con un real sentido de apoyo, tienen beneficios sobre la salud física, psíquica, emocional. De acuerdo con Orcasita y Uribe (2010) podría afirmarse que las investigaciones realizadas en cuanto al concepto de apoyo social tiene diversos efectos positivos con respecto a la salud y bienestar de los individuos, además de las investigaciones realizadas, las cuales demuestran que los sujetos que perciben altos niveles de apoyo social tienen un mayor autoconcepto, un estilo de afrontamiento más 
adecuado ante el estrés, una mayor autoestima y autoconfianza, mayor control personal y bienestar. Asimismo, Palomar y Cienfuegos (2007) señalan que el apoyo social brindado de una manera adecuada, puede considerarse como recurso necesario para la adaptación del sujeto a las exigencias medioambientales. De igual forma, dicho apoyo ayuda al individuo a disminuir las probabilidades de incurrir en conductas de riesgo como el consumo de drogas, alcohol, delincuencia y suicidio. De esta forma, los beneficios del apoyo social tienen repercusiones en todas las dimensiones de la vida de un ser humano, sin embargo, dichos beneficios se relacionan de forma directa con el tipo de apoyo social percibido de las personas que conforman su red de apoyo, que a su vez suministran los recursos al sujeto en cualquier etapa de su vida desde el neonato, pasando por el adolescente y llegando al adulto mayor. Por lo tanto, la importancia de las redes sociales no está en el número de personas que la integran sino en la calidad de apoyo, afecto y acompañamiento que reciben los adolescentes de estas (Gottlieb, 1998 citado por Guzmán et al., 2003).

\section{Conductas sexuales de riesgo en adolescentes}

Actualmente, las infecciones de transmisión sexual (ITS) entre ellas el virus de inmunodeficiencia humana (VIH), se presentan constantemente en la población adolescente; según registros publicados en el Plan nacional de respuesta ante el VIH y el Sida Colombia 20082011, se reportan que desde 1983 hasta 2007 se diagnosticó alrededor de 57500 personas con el virus y/o Sida, en los últimos ańos se ha logrado estimar que existe aproximadamente 171500 personas infectadas entre los 15 y 49 ańos. Acorde a estas cifras se estableció, que la población que en la cual se registra en $60 \%$ de los casos corresponde a los adolescentes y adultos jóvenes que oscilan entre los 15 y 34 años; de los cuales el $77 \%$ pertenece al género masculino (Ministerio de Protección Social, 2008). La infección por VIH/SIDA representan uno de los problemas de salud pública de mayor riesgo que ha tenido que afrontar el mundo en los últimos años; según Orcasita y Uribe (2010) 
los jóvenes y los adolescentes se han convertido en el grupo poblacional mas importante de vulnerabilidad ante este tipo de riesgos, pues ellos, inician sus relaciones sexuales, sociales, consolidan actitudes, valores y creencias que pueden incidir en conductas saludables o de riesgo.

Igualmente, una de las principales fuentes de riesgo en los adolescentes, que pueden desembocar en la adquisición de una ITS como el VIH/SIDA, suele ser la falta de búsqueda de fuentes de información sobre sexualidad en los grupos o sujetos como la familia, el sistema de salud o el colegio; generalmente los adolescentes suelen apoyarse durante esta etapa en el grupo de amigos, quienes habitualmente aún no poseen los recursos suficientes para ser guías; otra conducta que puede llevar a la adquisición del VIH en los adolescentes es la falta de conocimiento real de su pareja, junto con el deseo de experimentar e iniciar su vida sexual (Carvajal, 1998). Según la ENDS (2010) en Santander la edad promedio del inicio de relaciones sexuales en las mujeres es de $18.2 \%$; solo un $8 \%$ de las mujeres en unión utiliza el condón en sus relaciones sexuales, lo anterior significa que un $92 \%$ no toma ningún tipo de precaución contra las ITS.

Asociado a lo anterior, aparece en el adolescente el deseo de asumir riesgos sin proyectar las consecuencias futuras (Carvajal, 1998); lo que con lleva a utilizar conductas sexuales de riesgo (oro-genital y génito-anal) sin protección, bajo el efecto de sustancias psicoactivas relacionada con el consumo de drogas inyectables con agujas contaminadas, sostener relaciones sexuales con múltiples parejas sin protección, incluso en ocasiones con parejas no conocidas, lo cual aumenta el riesgo, así como los embarazos no planeados, e infecciones de transmisión sexual. De igual manera según ONUSIDA (2006), se debe tener presente la variable de vulnerabilidad, la cual se constituye como la capacidad de las personas para impedir ser infectados por el virus haciendo mención en tres factores; (a) factores personales, como falta de conocimientos y aptitudes necesarios para protegerse y proteger a otros; (b) factores relativos a la calidad y cobertura de los servicios, como inaccesibilidad debido a la distancia, costo y otros factores, y (c) factores sociales, como normas culturales y sociales, prácticas, creencias 
y leyes que estigmatizan e incapacitan a ciertas poblaciones y actúan como barreras para mensajes esenciales de prevención del VIH.

Otra investigación desarrollada por Vinaccia, Quiceno, Gaviria, Soto y Ballester (2007) afirman que uno de los aspectos relevantes en las conductas sexuales de riesgo de los adolescentes es la baja educación afectivo-sexual que les han brindado las redes de apoyo entre ellas el grupo primario e instituciones gubernamentales. Por otra parte, según Arias, Silva, Meneses, Castillo y Navarrete (2004), aseguran que en una población de 122 alumnos de un colegio de Bucaramanga, el $75 \%$ de ellos sostienen que iniciaron una vida sexual antes de los 16 años; entre tanto a diferencia de los adolescentes-hombres, las adolescentes-mujeres encuestadas aseguran que el inicio de su vida sexual, tendrá repercusiones para el futuro. Otra situación de riesgo puede estar propiciada no por los adolescentes, sino por los sujetos que forman parte de su sistema familiar y educativo, puesto que en los sistemas donde no existen recursos apropiados para el afrontamiento de periodos de transición, se da una restricción para el manejo de cualquier condición o suceso en momentos de crisis, poniendo en peligro al sujeto protagonista de la red. Por lo tanto, existen diversas causas que pueden llevar a los adolescentes a adoptar conductas de riesgo frente a su salud sexual y reproductiva. Se presentan factores personales, biológicos, heredados, factores ambientales y sociales que afectan e influyen en la aparición de dichos comportamientos; en ocasiones por la falta de adquisición de madurez durante esta etapa en el ciclo vital del ser humano, junto con la falencia de las redes de apoyo social (Alsinet, Pérez \& Agullo, 2003). No obstante, aunque el tema de las redes de apoyo social en los adolescentes es reciente en Colombia, las cifras expuestas en los diferentes estudios realizados por instituciones tanto oficiales como privadas evidencian una necesidad de abordar tanto las actitudes y comportamientos de riesgo que asumen los adolescentes, así como la influencia de sus redes próximas en la salud y bienestar del joven colombiano (Orcasita \& Uribe, 2010; Piña, 2004; y Arias et al., 2004). 


\section{Método}

La investigación que se llevó a cabo es de tipo descriptivo-correlacional, de corte transversal, la cual se caracteriza por "describir poblaciones sin que en su planteamiento se incluyan hipótesis propiamente dichas" (Montero \& León, 2005, p. 119), de esta manera, en este tipo de estudio se miden de forma independiente sus variables, para luego integrar dichas mediciones y decir cómo es y se manifiesta el fenómeno de interés. Dichas comparaciones implican una correlación más allá de lo puramente descriptivo, así de acuerdo con Montero y León (2005), el objetivo del tipo de estudio correlacional es medir el grado de relación existente entre dos variables.

\section{Participantes}

Se aplicó cuatro cuestionarios a 359 estudiantes adolescentes hombres y mujeres de los grados noveno, décimo y undécimo de una institución educativa pública del municipio de Lebrija en el departamento de Santander, con edades promedio entre los 12 y 18 años, la selección de cada uno de los sujetos se realizó por muestreo intencional.

\section{Instrumentos}

Se utilizaron cuatro instrumentos que miden las variables de apoyo social, conductas de riesgo y redes. A continuación se detallará en qué consiste cada uno de ellos:

1. Se utilizó el Cuestionario General diseñado por Uribe Rodríguez, Bermúdez y Buela-Casal (2005) citado por Uribe (2005), el cual recoge información específica de variables sociodemográficas tales como el estrato socioeconómico, el sexo, la edad, el tipo de institución educativa, el nivel de escolaridad, así como las personas con las que vive actualmente el joven. Asimismo, evalúa aspectos relacionados con las fuentes de información sobre la transmisión y las formas de prevención en comportamientos de riesgo. 
2. Se utilizó la encuesta sobre Conductas de Riesgo de Vigilancia (YRBS), desarrollado por el Centro De Control y Prevención de Enfermedades (CDC) (Kolbe, Kann y Collins, 1993), para monitorear la prevalencia de comportamientos de riesgo en jóvenes. Se utilizó el procedimiento metodológico de aplicación desarrollado por la Encuesta (CDC, 2000).

3. Para medir el Apoyo Social se utilizó el Cuestionario MOS de Apoyo Social, el cual permite indagar junto al apoyo global, otras cuatro dimensiones: 1) afectiva (demostración de amor, cariño y empatía); 2) de interacción social positiva (posibilidad de contar con personas para comunicarse); 3) instrumental (posibilidad de ayuda doméstica); y 4) emocional/informacional (posibilidad de asesoramiento, consejo, información). Tiene 20 ítems; el primero, encargado de investigar la red social, pregunta por el número de amigos íntimos y familiares cercanos que tiene el entrevistado. Los ítems restantes tienen una evaluación, mediante escala de tipo Likert puntuando de 1 (nunca) a 5 (siempre). (Martínez et al., 2004).

4. El APGAR Familiar, validado en poblaciones españolas (De la Revilla, Bailón \& Luna, 1991) determina el grado de satisfacción que percibe el encuestado con respecto a la función familiar; consta de 5 ítems que evalúan las cinco áreas diferentes en que los autores subdividen la función familiar: 1) adaptabilidad o capacidad de movilizar recursos; 2) cooperación o capacidad de participación; 3) desarrollo o capacidad de apoyar en la maduración física, emocional y la autorrealización; 4) afectividad o expresión de cariño; y, 5) capacidad de resolución o compromiso de dedicar tiempo a la familia. Cada pregunta se evalúa con un valor de 0 (casi nunca) a 2 (casi siempre) sobre una escala de Likert, obteniéndose al final un índice entre 0 y 10. Se clasifican las familias en normofuncionales $(>6)$ y disfuncionales $(\leq 6)$. 


\section{Procedimiento}

Se realizó el contacto con las directivas de la institución educativa y los participantes explicando los objetivos y alcances de la investigación, así como los aspectos éticos de anonimato y confidencialidad de la información. La aplicación del instrumento osciló entre 25 y 30 minutos. Los resultados fueron analizados en el paquete estadístico Statiscal Package for Social Sciences (SPSS) versión 17.0 para Windows.

\section{Resultados}

A continuación se muestran los datos obtenidos en la presente investigación. Inicialmente se realiza una descripción sociodemográfica de la muestra evaluada, posteriormente se realiza un análisis descriptivocorrelacional relacionado con la identificación de las conductas sexuales de riesgo, el tipo de apoyo social y funcionamiento familiar percibido, así como la relación entre dichas variables. Se utilizaron pruebas no paramétricas, puesto que la prueba de Kolmogorov-Smirnov de bondad de ajuste a la distribución normal mostró evidencias significativas de la no normalidad de los datos (p-valor $<5 \%)$. El nivel de significación utilizado fue del $5 \%$.

Se obtuvo información de 359 estudiantes de una institución pública del municipio de Lebrija, resultó una muestra homogénea en cuanto al sexo de los participantes, puesto que el $52.1 \%$ de los estudiantes son mujeres y el $47.9 \%$ son hombres. El $40.9 \%$ de los estudiantes es de estrato socioeconómico 2. En cuanto a la escolaridad de la población, la mayoría se concentra en los grados noveno y décimo. Los estudiantes encuestados reportan que viven con ambos padres (52.4\%) y solo un $2.2 \%$ vive solo con su padre. La mayoría de los estudiantes se identifican como heterosexuales $97.9 \%$, entretanto el $1.4 \%$ de los estudiantes reconoce que tienen una orientación bisexual y solo el $0.6 \%$ de los encuestados se identifican como homosexuales (Véase Tabla 1). 


\section{Tabla 1}

Caracterización sociodemográfica de los adolescentes

\begin{tabular}{lll}
\hline Sexo & Frecuencia & Porcentaje \\
\hline Masculino & 172 & 47.9 \\
Femenino & 187 & 52.1 \\
\hline Escolaridad & Frecuencia & Porcentaje \\
\hline Noveno & 139 & 39.0 \\
Décimo & 139 & 39.0 \\
Once & 78 & 21.9 \\
\hline Estrato & Frecuencia & Porcentaje \\
\hline Estrato 1 & 127 & 36.0 \\
Estrato 2 & 147 & 41.6 \\
Estrato 3 & 77 & 21.8 \\
Estrato 4 & 2 & 0.6 \\
\hline Orientación sexual & Frecuencia & Porcentaje \\
\hline Heterosexual & 324 & 97.9 \\
Bisexual & 5 & 1.5 \\
Homosexual & 2 & 0.6 \\
\hline Vives & Frecuencia & Porcentaje \\
\hline Padre & 8 & 2.3 \\
Madre & 107 & 30.7 \\
Ambos padres & 188 & 54.0 \\
Familiares & 41 & 11.8 \\
Amigos & 2 & 0.6 \\
Otras personas & 2 & 0.6 \\
\hline
\end{tabular}

El rango de edad de los estudiantes encuestados está entre 12 y 18 años, la edad promedio es de 15.25 años (Véase Tabla 2). 


\section{Tabla 2}

Estadísticos descriptivos para la edad

\begin{tabular}{cccc}
\hline Minimo & Máximo & Media & Desviación típica \\
\hline 12 & 18 & 15.25 & 1.170 \\
\hline
\end{tabular}

\section{Análisis descriptivo-correlacional}

El $66.7 \%$ de los estudiantes manifiestan haber obtenido información sobre conductas sexuales de riesgo, mientras que un 33.3\% manifiesta no haberla tenido (Véase Tabla 3).

\section{Tabla 3}

Información sobre conductas sexuales de riesgo

\begin{tabular}{ccc}
\hline ¿Ha recibido información? & Frecuencia & Porcentaje \\
\hline Sí & 238 & 66.7 \\
No & 119 & 33.3 \\
\hline
\end{tabular}

La Tabla 4 revela de dónde los jóvenes extraen la información sobre conductas sexuales de riesgo, se observa que el medio por el cual los adolescentes reciben mayor información es por las charlas en el colegio $59.3 \%$, seguido en su orden por sus familiares $52.4 \%$ y programas de televisión $52.4 \%$; el medio por el cual reciben poca información son las organizaciones gubernamentales $4.4 \%$, artículos de revistas $10.9 \%$ al igual que carteles en los buses $10.9 \%$, folletos distribuidos en la calle $14.1 \%$ y programas de radio $15.7 \%$ (Véase Tabla 4 ). 
Conductas sexuales de riesgo /Orcasita, Uribe, Castellanos y Gutiérrez Rodríguez

\section{Tabla 4}

Fuentes de información sobre prevención en conductas sexuales de riesgo

\begin{tabular}{lcc}
\hline Fuente & $N^{o}$ de respuestas & Porcentaje \\
\hline Programas Tv & 126 & $50.8 \%$ \\
Programas radio & 39 & $15.7 \%$ \\
Artículos revista & 27 & $10.9 \%$ \\
Artículos periódico & 40 & $16.1 \%$ \\
Carteles calle & 46 & $18.5 \%$ \\
Carteles buses & 27 & $10.9 \%$ \\
Amigos & 102 & $41.1 \%$ \\
Folletos colegio & 47 & $19.0 \%$ \\
Folletos calle & 35 & $14.1 \%$ \\
Internet & 109 & $44.0 \%$ \\
Profesionales & 113 & $45.6 \%$ \\
Charlas & 147 & $59.3 \%$ \\
Organizaciones & 11 & $4.4 \%$ \\
Folletos autoridades & 43 & $17.3 \%$ \\
Familiares & 130 & $52.4 \%$ \\
\hline
\end{tabular}

En cuanto a los temas de interés para los adolescentes; en el que desean obtener mayor información es sobre sexualidad 49.8\%, seguido su interés en saber sobre las enfermedades de trasmisión sexual 40.9\%; $\mathrm{VIH} /$ sida $38.0 \%$, consumo de drogas (33.2\%); siendo reportado por los jóvenes los temas de menor interés las píldoras para dietas o laxantes y la homosexualidad $7.7 \%$ respectivamente (Véase Tabla 5). 


\section{Tabla 5}

Temáticas de interés de los adolescentes

\begin{tabular}{lcc}
\hline Temas de interés & $N^{o}$ de respuestas & Porcentaje \\
\hline Tabaco & 22 & $7.0 \%$ \\
Fumar & 26 & $8.3 \%$ \\
Homosexualidad & 24 & $7.7 \%$ \\
Inhalantes & 26 & $8.3 \%$ \\
Alcohol & 98 & $31.3 \%$ \\
Sexualidad & 156 & $49.8 \%$ \\
Peso & 61 & $19.5 \%$ \\
Drogas & 104 & $33.2 \%$ \\
Abstinencia & 65 & $20.8 \%$ \\
Esteroides & 32 & $10.2 \%$ \\
VIH/Sida & 119 & $38.0 \%$ \\
Enfermedades & 128 & $40.9 \%$ \\
Depresión & 83 & $26.5 \%$ \\
Anticoncepción & 64 & $20.4 \%$ \\
Píldoras & 24 & $7.7 \%$ \\
Transgénero & 26 & $8.3 \%$ \\
Suicidio & 85 & $27.2 \%$ \\
Compartir & 25 & $8.0 \%$ \\
Dieta & 78 & $24.9 \%$ \\
Abuso & 63 & $20.1 \%$ \\
Perforaciones & 87 & $27.8 \%$ \\
\hline
\end{tabular}

\section{Comportamiento sexual de los adolescentes}

Teniendo en cuenta la información suministrada por los estudiantes se identificó que el $21.5 \%$ de ellos han tenido relaciones sexuales, de estos el $12.9 \%$ iniciaron su vida sexual entre los 15 y 16 ańos y el $7.0 \%$ a la edad de 13 o 14 años. Solo el $18.8 \%$ de las mujeres habían iniciado relaciones sexuales, mientras que un $38.8 \%$ de los hombres ha 
tenido relaciones sexuales, se encontró una relación estadísticamente significativa entre el sexo y el inicio de relaciones sexuales y la edad de inicio ( $\mathrm{p}$-valor $<5 \%$ ). En cuanto al número de personas con las cuales han tenido relaciones sexuales, el $15.5 \%$ sostiene haber tenido relaciones sexuales con una persona y el $6.2 \%$ con dos personas, también se encontró una relación significativa según el sexo ( $p$-valor=0.000). El 3.1\% de los adolescentes consumió bebidas alcohólicas o drogas antes de su última relación sexual; preocupantemente solo el $36.2 \%$ de la población sexualmente activa, la última vez que tuvo relaciones sexuales utilizó preservativo. El $0.6 \%$ de los adolescentes ha tenido relaciones sexuales tanto con hombres como con mujeres. La mayoría $73.5 \%$ de los adolescentes manifiestan tener un adulto en quien confiar y recibir un consejo en el momento que lo requiera, se evidenció que las mujeres en, mayor porcentaje que los hombres, tenían un adulto que le aconseje (p-valor=0.003) (Véase Tabla 6).

\section{Tabla 6}

Comportamiento sexual de los adolescentes

\begin{tabular}{lcccc}
\hline ¿Alguna vez ha tenido relaciones & $\begin{array}{c}\text { Hombre } \\
(\%)\end{array}$ & $\begin{array}{c}\text { Mujer } \\
(\%)\end{array}$ & $\begin{array}{c}\text { Total } \\
(\%)\end{array}$ & p-valor* \\
\hline Nexuales? & 58.8 & 77.9 & 72.8 & 0.000 \\
Nunca & 2.3 & 3.2 & 5.7 & \\
Sí & 38.8 & 18.8 & 21.5 & \\
\hline ¿Qué edad tenía cuando tuvo & Hombre & Mujer & Total & p-valor* \\
relaciones por primera vez? & $(\%)$ & $(\%)$ & $(\%)$ & \\
\hline Nunca he tenido relaciones sexuales & 61.2 & 81.2 & 71.6 & \multirow{2}{*}{0.000} \\
8 años de edad o menos & 3.5 & 1.1 & 2.2 & \\
9 o 10 ańos de edad & 4.7 & 0.0 & 2.2 & \\
11 o 12 ańos de edad & 4.7 & 0.0 & 2.2 & \\
13 o 14 ańos de edad & 8.2 & 5.9 & 7.0 & \\
15 o 16 ańos de edad & 16.5 & 9.7 & 12.9 & \\
17 años de edad o más & 1.2 & 2.2 & 1.7 & \\
\hline
\end{tabular}




\begin{tabular}{|c|c|c|c|c|}
\hline $\begin{array}{l}\text { Número de personas con las que ha } \\
\text { tenido relaciones sexuales }\end{array}$ & $\begin{array}{l}\text { Hombre } \\
(\%)\end{array}$ & $\begin{array}{l}\text { Mujer } \\
(\%)\end{array}$ & $\begin{array}{l}\text { Total } \\
(\%)\end{array}$ & p-valor* \\
\hline Nunca he tenido relaciones sexuales & 61.5 & 79.5 & 70.9 & 0.000 \\
\hline 1 persona & 14.8 & 16.2 & 15.5 & \\
\hline 2 personas & 10.7 & 2.7 & 6.5 & \\
\hline 3 personas & 5.9 & 0.5 & 3.1 & \\
\hline 4 personas & 3.6 & 0.0 & 1.7 & \\
\hline 5 personas & 1.2 & 0.5 & 0.8 & \\
\hline 6 o más personas & 2.4 & 0.5 & 1.4 & \\
\hline $\begin{array}{l}\text { ¿Consumió alcohol en su última } \\
\text { relación sexual? }\end{array}$ & $\begin{array}{l}\text { Hombre } \\
(\%)\end{array}$ & $\begin{array}{l}\text { Mujer } \\
(\%)\end{array}$ & $\begin{array}{l}\text { Total } \\
(\%)\end{array}$ & p-valor* \\
\hline Sí & 2.1 & 4.1 & 3.1 & 0.352 \\
\hline No & 97.9 & 95.9 & 96.9 & \\
\hline $\begin{array}{l}\text { ¿Usó usted o su pareja preservativo } \\
\text { durante su última relación sexual? }\end{array}$ & $\begin{array}{l}\text { Hombre } \\
(\%)\end{array}$ & $\begin{array}{l}\text { Mujer } \\
(\%)\end{array}$ & $\begin{array}{l}\text { Total } \\
(\%)\end{array}$ & p-valor* \\
\hline Sí & 41.4 & 28.6 & 36.2 & 0.071 \\
\hline No & 58.6 & 71.4 & 63.8 & \\
\hline $\begin{array}{l}\text { ¿Con quién ha tenido relaciones } \\
\text { sexuales? }\end{array}$ & $\begin{array}{l}\text { Hombre } \\
(\%)\end{array}$ & $\begin{array}{l}\text { Mujer } \\
(\%)\end{array}$ & $\begin{array}{l}\text { Total } \\
(\%)\end{array}$ & p-valor* \\
\hline Nunca he tenido relaciones sexuales & 59.6 & 83.3 & 72.0 & - \\
\hline Hombres & 4.8 & 15.6 & 10.4 & \\
\hline Mujeres & 34.9 & 0.6 & 17.1 & \\
\hline Hombres y mujeres & 0.6 & 0.6 & 0.6 & \\
\hline \multicolumn{5}{|l|}{ ¿Cuenta con un adulto que lo aconseje? } \\
\hline Sí & 66.3 & 80.3 & 73.5 & 0.003 \\
\hline No & 33.7 & 19.7 & 26.5 & \\
\hline
\end{tabular}

\section{Funcionamiento familiar en los adolescentes}

Según la información reportada de los adolescentes, el 67.8\% de ellos tiene una familia normofuncional y el $10.9 \%$ tiene una familia disfuncional y moderadamente disfuncional (21.3\%) (Véase Tabla 7). 


\section{Tabla 7}

Funcionamiento familiar reportado por el adolescente

\begin{tabular}{lcc}
\hline APGAR & \multicolumn{2}{c}{ Estudiantes } \\
& Frecuencia & Porcentaje \\
\hline Gravemente disfuncional & 38 & 10.9 \\
Moderadamente disfuncional & 74 & 21.3 \\
Normofuncional & 236 & 67.8 \\
\hline
\end{tabular}

El 70.1\% de las mujeres reportaron un funcionamiento familiar normal, mientras que en un porcentaje muy similar los hombres reportan lo mismo (Figura 1). No se encontró una relación significativa entre el funcionamiento familiar y sexo $(\mathrm{p}$-valor $=0.139)$, mediante la prueba chi-cuadrado de Pearson. También se encontró que no existe una asociación significativa en los adolescentes que han tenido relaciones sexuales o no, que consumen alcohol o no durante sus relaciones sexuales y que utilizan preservativos o no en sus relaciones sexuales con la variable funcionamiento familiar ( $\mathrm{p}$-valor $>5 \%$ ).

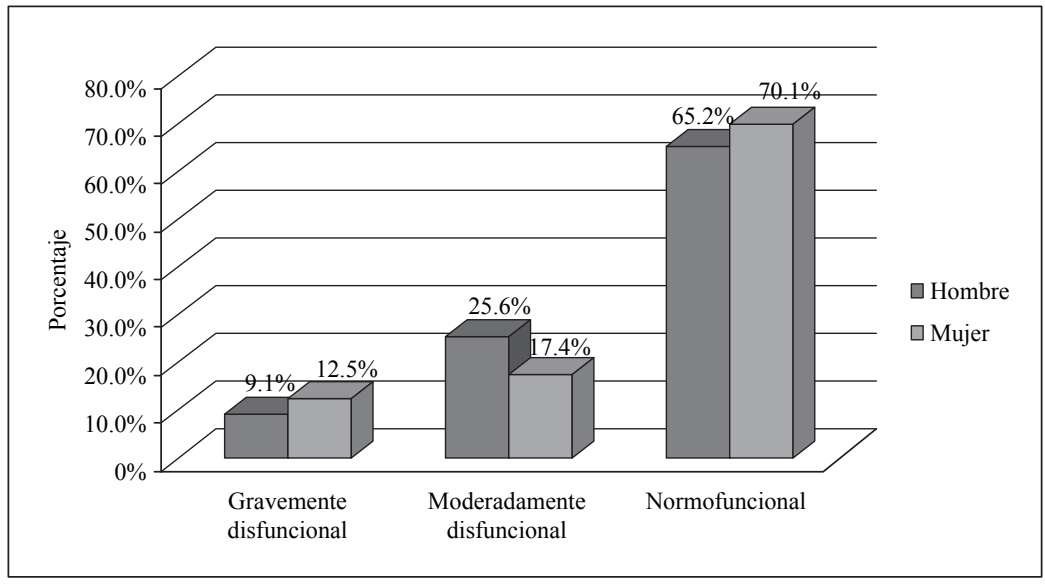

Figura 1. Funcionamiento familiar en función del sexo 


\section{Tipo de apoyo social percibido}

Puntuaciones elevadas en el cuestionario de apoyo social percibido evidencian un mayor apoyo percibido y un puntaje bajo representa un menor apoyo social percibido. En la Tabla 8 se muestran algunas estadísticas descriptivas para las puntuaciones de las subescalas, se observa que todas las dimensiones sobrepasan el punto medio de la escala, es decir, que los adolescentes logran percibir un buen apoyo social. Los estudiantes perciben apoyo emocional, instrumental, interacción social y afectiva de sus redes de apoyo.

\section{Tabla 8}

Estadisticas descriptivas para el cuestionario de apoyo social percibido

\begin{tabular}{lccccc}
\hline MOS & Mínimo & Máximo & $\begin{array}{c}\text { Punto } \\
\text { Medio }\end{array}$ & Media & Desv. típ. \\
\hline Apoyo emocional & 8 & 40 & 24 & 32.41 & 7.23 \\
Apoyo instrumental & 5 & 20 & 12.5 & 17.55 & 2.98 \\
Interacción social & 7 & 20 & 12.5 & 16.92 & 3.28 \\
Apoyo afectivo & 4 & 15 & 9 & 13.06 & 2.44 \\
\hline
\end{tabular}

Al comparar el apoyo social percibido en función del sexo, solo se encontró una diferencia significativa mediante la prueba de Mann-Whitney en el apoyo emocional de los hombres y las mujeres ( $\mathrm{p}$-valor=0.006), y se evidenció que las mujeres recibían un apoyo emocional mucho más alto que los hombres (figura 2).

Con respecto a las redes de apoyo, los adolescentes piden ayuda con mayor frecuencia a la familia (53.7\%), seguida de los amigos (38.8\%) (Véase Tabla 9). En promedio los adolescentes cuentan con cuatro personas en sus redes de apoyo íntimas (amigos y familiares) (Véase Tabla 10). 


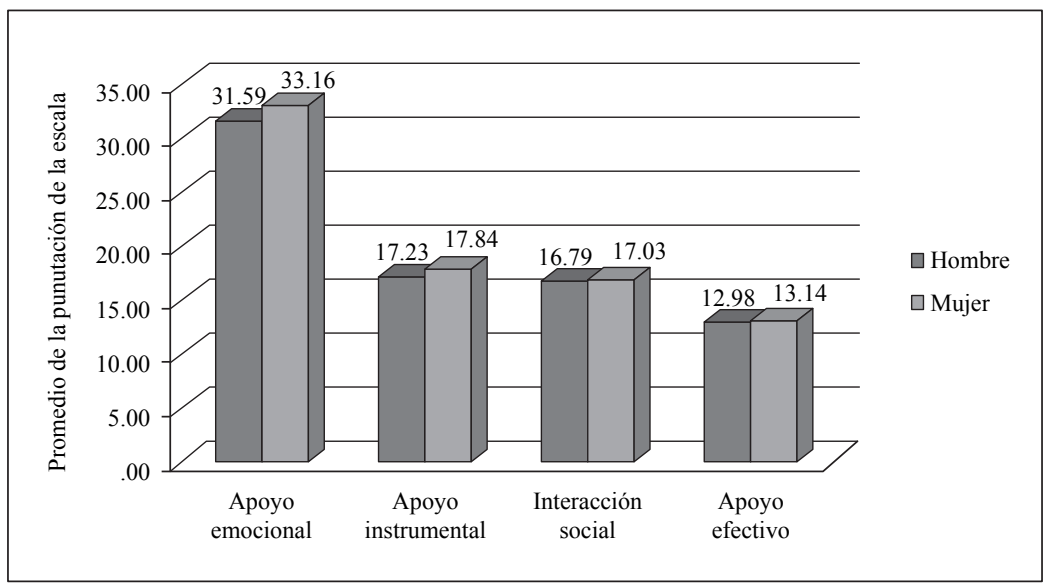

Figura 2. Apoyo social percibido en función del sexo

\section{Tabla 9}

Frecuencia de red de ayuda que solicitan los adolescentes

¿A quién pide usted ayuda con mayor frecuencia)

Estudiantes

\begin{tabular}{lcc}
\hline Familia & 173 & 53.7 \\
Amigos & 125 & 38.8 \\
Otras personas & 24 & 7.5 \\
\hline
\end{tabular}

\section{Tabla 10}

Número de redes intimas de apoyo social

\begin{tabular}{lcccc}
\hline Redes de apoyo íntimas & Mínimo & Máximo & Media & Desv. típ. \\
\hline Amigos & 0 & 20 & 4.20 & 3.659 \\
Familiares & 0 & 20 & 4.21 & 4.161 \\
\hline
\end{tabular}




\section{Apoyo social percibido y conductas sexuales de riesgo}

Para comprobar si el apoyo social percibido difiere en función de los comportamientos sexuales de riesgo que adoptan los adolescentes, se realizó un análisis comparativo. Bajo un nivel de significación del $5 \%$, con la prueba no paramétrica de Mann-Whitney, se encontraron diferencias significativas entre los adolescentes que han iniciado sus relaciones sexuales y los que no han iniciado en cuanto al apoyo emocional ( $\mathrm{p}$-valor $=0.02$ ) e instrumental ( $\mathrm{p}$-valor=0.001), es decir, que los adolescentes que iniciaron su vida sexual perciben un apoyo emocional e instrumental, mucho más bajo que los adolescentes que no han iniciado su vida sexual (Véase Tabla 11). No se encontraron diferencias significativas en función del uso del preservativo y consumo de alcohol durante las relaciones sexuales.

\section{Discusión}

El apoyo social y las conductas de riesgo, son aspectos importantes para comprender la salud sexual y reproductiva los adolescentes; los resultados obtenidos en la presente investigación, logran identificar y analizar algunos factores relacionados con los comportamientos de riesgo, que son reportados por Orcasita y Uribe (2010), Pińa (2004) y Arias et al. (2004) en investigaciones previas realizadas con adolescentes colombianos frente al VIH, sin abordar la variable apoyo social.

El apoyo social es una temática que ha logrado alcanzar gran importancia dentro del campo investigativo debido a que esta trae beneficios en el desarrollo de los individuos, desde hace poco tiempo esta temática se ha incorporado dentro del contexto colombiano, lo que genera que en la actualidad hayan algunos referentes investigativos orientados en la población adolescente específicamente. Con respecto a la ciudad de Bucaramanga y su área metropolitana aunque se han realizado acercamientos investigativos con adolescentes Piña (2004) y Arias et al. (2004), hasta ahora las investigaciones no se ha centrado en la identificación de las conductas sexuales de riesgo y el apoyo social percibido por adolescentes escolarizados. 


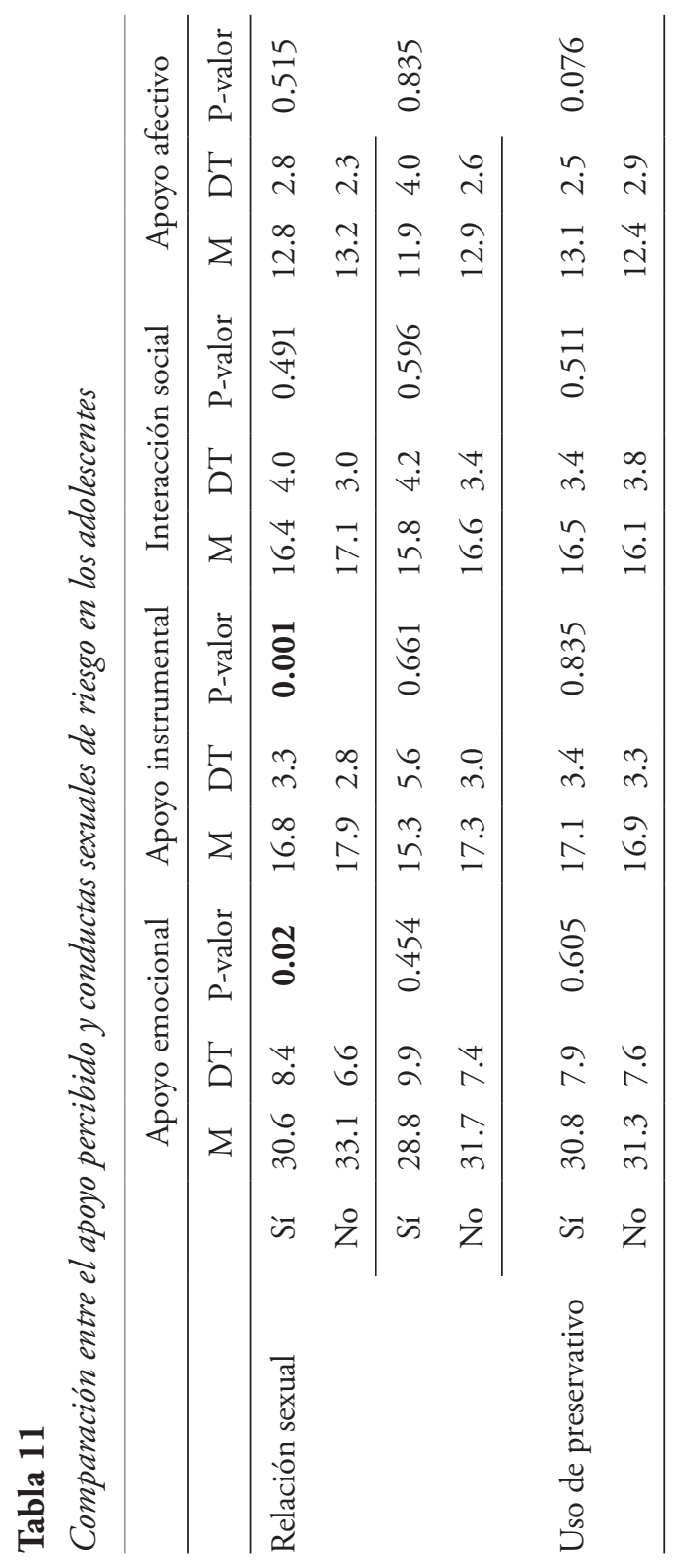




\section{Discusión}

El apoyo social y las conductas de riesgo, son aspectos importantes para comprender la salud sexual y reproductiva los adolescentes; los resultados obtenidos en la presente investigación, logran identificar y analizar algunos factores relacionados con los comportamientos de riesgo, que son reportados por Orcasita y Uribe (2010), Pińa (2004) y Arias et al. (2004) en investigaciones previas realizadas con adolescentes colombianos frente al VIH, sin abordar la variable apoyo social.

El apoyo social es una temática que ha logrado alcanzar gran importancia dentro del campo investigativo debido a que esta trae beneficios en el desarrollo de los individuos, desde hace poco tiempo esta temática se ha incorporado dentro del contexto colombiano, lo que genera que en la actualidad hayan algunos referentes investigativos orientados en la población adolescente específicamente. Con respecto a la ciudad de Bucaramanga y su área metropolitana aunque se han realizado acercamientos investigativos con adolescentes Piña (2004) y Arias et al. (2004), hasta ahora las investigaciones no se ha centrado en la identificación de las conductas sexuales de riesgo y el apoyo social percibido por adolescentes escolarizados.

El análisis de los datos arrojados en la presente investigación permite corroborar que existe relación entre el apoyo social y las conductas sexuales de riesgo como señala Felton y Shin, 1992 citados por Barrón (1996), en el que se describe el apoyo social como un conjunto de recursos con el que puede llegar a contar un sistema, grupo familiar y/o individuo a lo largo de su vida y que ocasionalmente puede servirle de soporte en momentos de crisis para llegar a niveles de asimilación, acomodación y superación en situaciones que generan tensión. Cabe resaltar que los jóvenes que han iniciado su vida sexual perciben un menor apoyo social, en comparación con los adolescentes que no han iniciado la vida sexual, lo cual aumenta el riesgo a que esta población busque fuentes de información y/o apoyo no apropiados para despejar dudas que se pudieran presentar, al igual que recibir orientaciones en cuanto a la salud sexual u otras situaciones que tuviera que afrontar el adolescente. 
Uno de los objetivos centrales de la investigación fue evaluar el apoyo social percibido y las conductas sexuales de riesgo que presentan los adolescentes del Lebrija-Santander, se encontró que los adolescentes han recibido información sobre conductas sexuales de riesgo; dichos aportes principalmente se originan en charlas dadas por la institución educativa, los familiares y los programas de televisión. Por consiguiente es importante que las entidades especialistas en los programas de salud sexual y reproductiva de los adolescentes colombianos, conformen junto con los medios de comunicación masivos, grupos interdisciplinarios (médicos, psicólogos, comunicadores sociales, pedagogos, entre otros) que construyan lineamientos para diseñar programas acordes a las necesidades de los adolescentes y las temáticas de interés sobre sexualidad reportadas en la presente investigación, donde se orienten y regule la información recibida. Además sería apropiado vincular a las principales redes de apoyo social identificadas con el fin de fortalecerlas para que estas establezcan estrategias que lleven a una adecuada orientación sexual a los jóvenes.

Asimismo, la familia es una de las primeras redes de apoyo social, con que cuenta los seres humanos, ya que es la encargada de proveer emocional y materialmente a los individuos, en sus primeras etapas de desarrollo. Esta ejerce una función indispensable para el proceso de socialización, debido a que por medio del ejemplo otorga herramientas que garantiza a los adolescentes la inclusión en grupos sociales, adaptación a las normativas de las instituciones educativas y el afianzamiento entre sus iguales (Valdés, 2007); pero el vínculo entre los adolescentes y la familia va más allá de las relaciones de consanguinidad que pudieran tener, esta relación entre familia y adolescente está enmarcada por los lazos emocionales que se establecen entre los miembros y se originan desde los canales de comunicación asertiva, dinámica familiar y la expresión de sentimientos entre unos y otros (Mendizabal y Anzuares, 1999). Lo cual se ve reflejado en los datos arrojados en esta investigación, ya que se identificó que los adolescentes de esta institución, conciben como primera fuente de apoyo a la familia debido a que recurren a ella con el fin de obtener información continua en varios momentos sobre cuestionamientos generadores de dudas; asimismo, 
se resalta que los adolescentes que han iniciado su vida sexual perciben un menor apoyo emocional frente a los adolescentes que no tienen una vida sexualmente activa.

Por otra parte se encontró que aunque los jóvenes reciben información, principalmente por los medios visibles y auditivos, estos presentan dudas y/o bajo conocimiento sobre sexualidad, ITS, VIH/ sida, lo cual es importante resaltar, ya que a diferencia de otras etapas de desarrollo en la adolescencia se evidencia el interés y la necesidad de recibir mayor información sobre temas de sexualidad. Por consiguiente se hace necesario investigar las ideas erróneas o falsas concepciones que los jóvenes tienen sobre estas temáticas donde las redes de apoyo brinden adecuada información y generen estrategias de autocuidado, obteniendo así mayores niveles de protección en la práctica de conductas sexuales de riesgo. Vinaccia et al. (2007) afirman que uno de los aspectos relevantes en las conductas sexuales de riesgo es la baja educación afectivo-sexual que las redes (grupo primario e instituciones gubernamentales) han brindado a los jóvenes.

Una de las responsabilidades que hoy en día tienen las redes de apoyo de los adolescentes es reestructurar esquemas cognitivos, paradigmas y/o conceptos inadecuados existentes sobre la sexualidad e instruir con información apropiada y veraz a los jóvenes para otorgarles las herramientas adecuadas que permitan que estos adopten una actitud responsable tanto para consigo mismos como para sus parejas. Aunque en la actualidad los diferentes medios de comunicación, algunas páginas de internet y la información suministrada por los pares, mencionan temáticas relacionadas a la sexualidad, métodos anticonceptivos, número de parejas sexuales, infecciones de transmisión sexual, entre otros; muchas veces la información recibida de estas fuentes no es verdadera o no es sustraída de entidades y/o profesionales especialistas en la salud sexual y reproductiva, lo cual lleva a aumentar los factores de riesgo existentes para esta población. Es alarmante observar el aumento que año tras año reporta el Observatorio de Salud Pública de Santander en lo concerniente a las personas infectadas de VIH en Bucaramanga y su área metropolitana (Observatorio de Salud Pública de Santander, 2010). 
Lo cual, es contrastado con las conductas sexuales de riesgo que adoptan las mujeres colombianas entre los 15 y 40 años de edad (ENDS, 2010) y lo corroborado en esta investigación sobre el riesgo existente entre los jóvenes de 15 y 16 años que han iniciado su vida sexual.

De igual forma, los datos obtenidos por Mosquera y Mateurs (2003) citados por Orcasita, Valderrama y Uribe (2010) evidencian que a pesar que los jóvenes reciben información concreta sobre planificación y prevención del VIH continúan con conocimientos inadecuados, lo cual llevó a determinar la importancia de reforzar la educación a través de la televisión; puesto que al igual que esta investigación hace parte de una de las fuentes más relevantes de información.

La edad de inicio de las relaciones sexuales, es determinante para el desarrollo físico, social, emocional y psicológico de los adolescentes; de la misma forma, esta conducta está enmarcada por factores ambientales, personales, culturales y sociales en que se desarrollan los adolescentes. Aunque este suceso hace parte del desarrollo del ciclo vital, es importante que los individuos cuenten con una madurez física, cognitiva y emocional que los lleve a contemplar la responsabilidad y la incidencia que trae el inicio de las relaciones sexuales en los adolescentes (Alsinet, Pérez \& Agullo, 2003; Campo et al., 2004). Los resultados obtenidos en la presente investigación que se relacionan con la edad de inicio de las relaciones sexuales, es similar a los rangos encontrados por Campo et al. (2004) y Orcasita y Uribe (2010).

Dado que se estableció que el rango de edad más frecuente en que los adolescentes iniciaron su vida sexual fue entre los 15 a 16 ańos de edad y una investigación realizada en una población de estudiantes de un colegio de Bucaramanga, con características sociodemográficas similares reportaron un rango similar en el inicio de las relaciones sexuales, a diferencia de los datos arrojados por el estudio realizado en jóvenes diagnosticados y no diagnosticados con VIH/SIDA, donde el promedio de inicio de las relaciones sexuales del grupo de diagnosticados fue inferior en comparación con los adolescentes evaluados (Campo et al., 2004; Orcasita \& Uribe, 2010). Por tanto es necesario que los programas de prevención establecidos por entidades gubernamentales 
y educativas, vinculen a individuos con edades más tempranas, con la intención que estos preadolescentes cuenten con herramientas apropiadas para asumir la vida sexual y la adolescencia

Acorde a los datos arrojados, un porcentaje importante de la población ha iniciado su vida sexual bajo la exposición a conductas de riesgo, debido a que la mayoría de los adolescentes no utilizaron el preservativo, además de haber ingerido alcohol antes de tener su última relación sexual. Es preocupante este tipo de conductas asumidas por los jóvenes, puesto que según los datos publicados por el Ministerio de Protección Social (2008), es precisamente esta población en la que se registran el mayor número de personas infectadas por el VIH. Es así como se puede determinar que las conductas de riesgo más frecuentes en los adolescentes están relacionadas con el inicio de la vida sexual, consumo de sustancias psicoactivas y un bajo uso del preservativo durante las relaciones sexuales ya sean de tipo vaginal, oral o anal. Dentro de diversas investigaciones los factores que se han identificado son similares a los reportados en este estudio (Carvajal, 1998; Palacios, Bravo \& Andrade, 2007) se requiere canales de comunicación que generen en los adolescentes acompañamiento y orientaciones sobre la sexualidad, riesgos, enfermedades y métodos de planificación familiar, generados principalmente en la familia nuclear, instituciones educativas, entidades prestadoras de servicios médicos, entre otros (Herrera, 1999).

En lo concerniente al ámbito de la amistad, para los adolescentes sus pares cuentan con un papel bastante significativo, ya que son estos los que adquieren protagonismo en el proceso de desarrollo y la iniciación a la vida sexual (Fernández, 2000). Dado que la relación y consolidación de compańeros a amigos logra que se establezca una sensación de apoyo, seguridad y acompańamiento en momentos críticos (Orcasita \& Uribe 2010).

Por otra parte el deseo de asumir constantes retos en los adolescentes y el nivel de influencia del grupo puede resultar ser determinante en el desarrollo de los jóvenes (Carvajal, 1998). Por tanto, como se ha mencionado anteriormente, es vital que los adolescente cuenten con redes de apoyo que no sean exclusivamente su soporte material como 
dinero, comida, vivienda, entre otros (Méndez \& Barra, 2008) sino cuenten con una orientación basada en el respeto por el otro, manifestación de sentimientos, afectos, pensamientos, reconocimiento, escucha y crecimiento de la autoestima, más aún se han iniciado su vida sexual, ya que estos adolescentes necesitan mayores recursos los cuales son suministrados por las redes de apoyo social.

Uno de los pilares fundamentales del ser humano es la familia, en particular para los adolescentes, ya que como se ha expuesto es una etapa de cambios que requieren de guía y acompañamiento constante. Dentro del proceso de evaluación se evidenció que las redes de apoyo de los adolescentes que iniciaron la vida sexual presentan dificultades en la transmisión de herramientas de autocuidado, utilización de canales de comunicación asertivos y en la minimización de riesgos los cuales afectan no solo al adolescente y su familia sino a la sociedad en general.

Finalmente es necesario destacar que de acuerdo a los datos arrojados en la investigación es necesario que los profesionales en la salud de las distintas disciplinas contribuyan de manera activa con el aumento en la calidad de vida y un adecuado desarrollo de los adolescentes, creando así programas de intervención que fortalezcan estas redes de apoyo al igual que en las instituciones educativas, a los educadores encargados de los programas de prevención, para que estos brinden información adecuada y oportuna frente a las conductas de riesgo identificadas en la presente investigación.

La red de apoyo más significativa con la que cuenta este grupo de jóvenes es la familia; es recomendable que ellos junto con los educadores asuman un papel mas activo, vinculándose o conformando con la orientación de los profesionales pertinentes, programas interdisciplinarios de salud sexual y reproductiva, con el objetivo de prevenir conductas de riesgo que atentan contra la salud y el bienestar de la población estudiantil. Dado que la mayor parte de estos estudiantes vive con ambos padres (papá y mamá) o uno de ellos, es necesario que sean fuentes de apoyo adecuadas, los cuales brinden consejos, información, apoyo continuo de acuerdo a las necesidades del adolescente; asimismo, tratar temas con naturalidad y así proporcionar pautas 
de crianza acertadas donde ayuden a sus hijos con sus logros y al mismo tiempo a aquellas dificultades que esta etapa puede acarrear.

Dentro de las instituciones gubernamentales creadas con el objeto de mejorar la calidad de vida de la población colombiana y en especial la de los adolescentes, es importante que se realice un acompańamiento u orientación a los padres de familia para estos cuenten con las herramientas suficientes e información actualizada, con el fin de generar entre padres e hijos la confianza de hablar sobre temas de salud sexual, métodos anticonceptivos, infecciones de transmisión sexual, autocuidado, sustancias psicoactivas, para que los padres de familia se conviertan en las principales fuentes de apoyo real y confiables para los adolescentes.

Teniendo en cuenta la información suministrada por los adolescentes es necesario que desde la familia y la academia se empodere a los jóvenes basando sus estilos de vida en el autocuidado, autoestima y en especial en la capacidad de decir NO, cuando alguna decisión pueda llegar a atentar su integridad, así como buscar ayuda en las redes de apoyo establecidas por los jóvenes. Asimismo, los medios de comunicación como la radio, prensa, revistas y principalmente la televisión deben servir de apoyo a todos y cada uno de estos programas, con el fin de llenar a la población del mayor conocimiento respecto a esta temática; donde sean los programas de televisión la otra fuente de información sobre conductas sexuales de riesgo (prevención, trasmisión y tratamiento).

Finalmente este tipo de investigaciones puede generar aportes a nivel académico e investigativo, en los profesionales de la salud, con el fin de desarrollar y ejecutar modelos de intervención con los jóvenes adolescentes escolarizados donde se describan cuáles son las redes de apoyo usándolas como principal factor de prevención ante las conductas de riesgo, proporcionando beneficio a los adolescentes y por ende se logre entre toda la población un trabajo continuo y participativo. Asimismo, que a través de este tipo de investigaciones se puedan realizar programas y proyectos donde las instituciones (gubernamentales y educativas) se vinculen de manera directa, junto con la comunidad a fortalecer conocimientos, actitudes y prácticas que beneficien la salud sexual y reproductiva de cada uno de los jóvenes. 


\section{Referencias}

Alsinet, C., Pérez, R. M y Agullo, M. J. (2003). Adolescentes y percepciones del riesgo. Jóvenes. Revista de estudio sobre juventud, 18, 90-101.

Andersen, H. (1999). Conversación, lenguaje y posibilidades. Un enfoque posmoderno de la terapia. Buenos Aires: Amorrortu.

Arias, A., Silva, J. L., Meneses, M., Castillo, M. y Navarrete, P. A. (2004). Factores asociados con el inicio temprano de relaciones sexuales en estudiantes de un colegio de Bucaramanga, Colombia. Revista de Psiquiatría, 32(4), 367-377.

Avendaño, M. J. \& Barra, E. (2008). Autoeficacia, apoyo social y calidad de vida en adolescentes con enfermedades crónicas. Terapia Psicológica, 26(2), 162-175.

Barrón, A. (1996) Apoyo Social Aspectos Teóricos y aplicaciones. Madrid: Siglo XXI de España Editores S. A.

Belzaa, M., Clavoc, P., Ballesteros, J., Menéndez, B. L., Castilla, J., Sanz, S., Jerez, N., Rodríguez, C., Sánchez, Fl. \& del Romero, J. (2004) Condiciones sociolaborales, conductas de riesgo y prevalencia de infecciones de transmisión sexual en mujeres inmigrantes que ejercen la prostitución en Madrid. Gaceta Sanitaria, 18(3), 177-183.

Campo-Arias, A., Silva, J., Meneses, M., Castillo, M. \& Navarrete,

P. (2004) Factores asociados con el inicio temprano de relaciones sexuales en estudiantes adolescentes de un colegio de Bucaramanga, Colombia. Revista Colombiana de Psiquiatria, 33(4), 367-377.

Cañellas, S., Pérez, J., Noguer, I., Villaamil, F., García, L., De la Fuente, L., Belza J. \& Castilla, J. (2000). Conductas sexuales de riesgo y prevalencia de infección por VIH en hombres con prácticas homosexuales y bisexuales en la comunidad de Madrid. Revista Española de Salud Pública, 74(1), 25-32.

Carvajal, G. (1998). Adolecer la aventura de una metamorfosis. Madrid: Editorial Tiresias. 
Center for Disease Control and Prevention. (2000). Cluster of HIV infected adolescents and young adults - Mississippi. Morbidity and Mortality Weekly Report, 49, 861-64.

De La Reviia L, Bailón, E., De Dios Luna, J., Delgado A., Prados M., Freitas, L. (1991). Validación de una escala de apoyo social funcional para su uso en la consulta del médico de familia. Aten Primaria, 8, 688-92.

Fernández, L. (2000). Instituciones educativas. Madrid: Editorial Paidós. Guzmán, J.M.; Huenchuan, S. \& Montes, V. (2003). Redes de apoyo de personas mayores: Marco Teórico conceptual. Ponencia presentada en el simposio Viejos y Viejas. Participación, Ciudadanía e Inclusión Social 51 Congreso Internacional de Americanistas, 1-20.

Herrera, P. (1999). Principales factores de riesgo psicológicos y sociales en el adolescente. Revista cubana de Pediatría, 71(1), 39-42.

Juárez, C., Valdez, R. \& Hernández, D. (2001). Las redes sociales y la nocion de apoyo social en mujeres con experiencia de violencia conyugal. Revista Hispana para el Análisis de Redes Sociales. 1-10.

Kolbe, L. J., Kann, L. \& Collins, J. L. (1993). Overview of the Youth Risk Behavior Surveillance System, Public Health Reports, 108 (Suppl 1), 2-10.

Martin, E. \& Dávila, L. (2008). Redes de apoyo social y adaptación de los menores en acogimiento residencial. Psicothema, 20(2), 229-235.

Martínez, R., Pinzón, P., Maiquez, A., Herrera, J., De Benito, M. \& Cuesta, E. (2004). ¿Tienen apoyo social y familiar los drogodependientes que participan en el programa "libre de drogas" en prisión? Medicina de Familia, 5(1), 16-26.

Mendizabal, J. A., Anzuares, B. (1999). La Familia y Adolescentes. Revista Médica General Hospital General de México, 63(3), 191-197.

Méndez, P y Barra, E. (2008). Apoyo Social Percibido en Adolescentes Infractores de Ley y no Infractores. Psykhe 17(1), 59-64.

Ministerio de Protección Social (2008). Plan nacional de respuesta ante el VIH y el Sida. Colombia 2008-2011. Bogotá. 
Minuchin, S y Fishman, H. (1983). Familias (pp. 25-40). Barcelona: Paidós Ibérica S. A.

Morales, F., Cerezo, M., Fernández, F., Infante, L. \& Trianes, M. (2009). Eficacia de una intervención para incrementar apoyo social en adolescentes discapacitados motores a partir del voluntariado de estudiantes de educación secundaria. Revista Latinoamericana de Psicología, 41(1), 141-150.

Musitu, G. \& Cava, M. (2003). El rol del apoyo social en el ajuste de los adolescentes. Intervención Psicosocial, 12(2), 179-192.

Observatorio de Salud Pública de Santander. (2010). Celebración día mundial de la lucha contra el Sida. Recuperado en abril 8 de 2011. Recuperado de: http://www.saludsantander.gov.co/web/ index.php?option=content $\& v i e w=$ article $\& i d=109$ :secretariade-salud-departamental-se-vincula-a-los-actos-de-celebraciondel-dia-mundial-de-la-lucha-contra-el-sida\&catid $=7=$ secretariade-la-salud-de-santander\&ltemid=5

ONUSIDA (2010). Informe de ONUSIDA sobre la epidemia mundial de sida 2010. Recuperado 8, abril, 2011 http://www.unaids. org/globalreport/Global_report_es.htm

Orcasita, L.T. \& Uribe, A. F. (2010). La importancia del Apoyo Social en el Bienestar de los Adolescentes. Psychologia, Avances de la disciplina. 4(2), 69-82.

Orcasita, L.T., Peralta, A., Valderrama, L. \& Uribe, A. F. (2010). Apoyo social y conductas de riesgo en adolescentes diagnosticados y no diagnosticados con VIH/SIDA en Cali-Colombia. Revista Virtual Universidad del Norte, 30, 155-195.

Palacios, D., Bravo, F. \& Andrade, P. (2007). Consumo de alcohol y conducta sexual de riesgo en adolescentes. Psichology International, 18 (4), 1-13.

Palomar, J. y Cienfuegos, Y. (2007). Pobreza y Apoyo Social: Un estudio comparativo en tres niveles socioeconómicos. Revista Interamericana de Psicología, 41(2), 177-188.

Pardo, G., Sandoval, A. \& Umbarita, D. (2004). Adolescencia y depresión. Revista Colombiana de Psicología, 13, 13-28. 
Piña, J. (2004). Eventos disposicionales que probabilizan la práctica de conductas de riesgo para el VIH/SIDA. Anales de Psicología, 20(1), 23-32.

Profamilia (2010). Encuesta nacional de Demografía y Salud ENDS. Recuperado en abril 8 de 2011 de http://encuestaprofamilia. com/documentos/boletines/departamentales/santander.pdf

UNGASS (2010). Seguimiento de la declaración de compromiso sobre el VIH/sida. Informe nacional república de Colombia. 1-146. Recuperado en julio 16 de 2011 de http://latincial.net/PDF/ colombia_2010_country_progress_report_es.pdf

Uribe, A. F. (2005). Evaluación de factores psicosociales de riesgo para la infección por el VIH/SIDA en adolescentes colombianos. Tesis Doctoral. Granada (España): Universidad de Granada, Departamento de Personalidad, Evaluación y Tratamiento Psicológico.

Uribe, A. F., Orcasita, L. T. \& Vergara, T. (2010). Factores de riesgo para la infección por VIH/sida en adolescentes y jóvenes Colombianos. Acta Colombiana de Psicología, 13(1), 11-24.

Valdés, A. (2007). Familia y desarrollo. Intervención en terapia familiar. Bogotá: Editorial Manual Moderno S.A.

Vinaccia. S., Quiceno. M., Gaviria. A., Soto. A., Gil, M. \& Ballester, R. (2007). Conductas Sexuales de Riesgo para la Infección por VIH/Sida en Adolescentes Colombianos. Terapia Psicológica, 25(1), 39-50.

Recibido: 02 de febrero de 2012 Aceptado: 13 de octubre de 2012 